\title{
Publisher Correction: The MHC-II peptidome of pancreatic islets identifies key features of autoimmune peptides
}

Xiaoxiao Wan, Anthony N. Vomund, Orion J. Peterson, Alexander V. Chervonsky, Cheryl F. Lichti (iD and Emil R. Unanue (i) Correction to: Nature Immunology https://doi.org/10.1038/s41590-020-0623-7, published online 9 March 2020.

In the version of this article initially published, in Fig. 3f, right panel, the labels for the Ins1C:51-61 and Ins1C:51-61E peptide EC fata were switched. The white data points correspond to Ins1C:51-61E, and the blue data points correspond to Ins1C:51-61. The error has been corrected in the HTML and PDF versions of the article.

Published online: 1 April 2020

https://doi.org/10.1038/s41590-020-0670-0

(c) The Author(s), under exclusive licence to Springer Nature America, Inc. 2020 